\title{
Physical exercise and functional fitness
}

\section{in independently living vs institutionalized elderly women: a comparison of 60- to 79-year-old city dwellers}

\author{
This article was published in the following Dove Press journal: \\ Clinical Interventions in Aging \\ 24 April 2015 \\ Number of times this article has been viewed
}

\author{
Hélio L Furtado' \\ Nelson Sousa ${ }^{2}$ \\ Roberto Simão 3 \\ Fabio Dutra Pereira' \\ José Vilaça-Alves² \\ 'Castelo Branco University, Rio de \\ Janeiro, Brazil; ${ }^{2}$ Research Center \\ for Sport, Health, and Human \\ Development, University of Trás-os- \\ Montes and Alto Douro, Vila Real, \\ Portugal; ${ }^{3}$ School of Physical Education \\ and Sports, Rio de Janeiro Federal \\ University, Rio de Janeiro, Brazil
}

Purpose: To compare functional fitness (FF) levels among independent-living (IL) and day care (DC) elderly women of different age groups and to analyze changes in FF after 8 months of participation in an exercise program intervention for the IL elderly women.

Materials and methods: A total of 674 elderly women were divided into four IL groups with age in the range of 60-64 years (IL60-64, $n=149$ ), 65-69 years (IL65-69, $n=138$ ), 70-74 years (IL70-74, $n=135$ ), and 75-79 years (IL75-79, n=83), and four DC groups with age in the range of 60-64 years (DC60-64, n=35), 65-69 years (DC65-69, n=34), 70-74 years (DC70-74, n=47), and 75-79 years (DC75-79, $\mathrm{n}=53$ ). The intervention consisted of a multimodal exercise training, 3 days per week for 8 months. Senior Fitness Test battery performances were obtained at baseline and after 8-month intervention.

Results: Significant differences were identified between all IL groups and DC groups in all FF tests $(P<0.001)$, except between IL70-74 and DC70-74 in the chair sit-and-reach. Analysis of covariance (ANCOVA) identified significant improvements in all FF tests between pre- and posttests in the IL groups $(P<0.001)$, except in the chair sit-and-reach for the IL70-74. ANCOVA also showed a significant declining performance in all FF tests for DC groups $(P<0.001)$, except in the chair sit-and-reach for the DC70-74 and DC75-79.

Conclusion: IL women are more fit than institutionalized DC elderly women. The multimodal training was effective in improving all FF components related to daily living activities, in all age groups. In contrast, institutionalized elderly showed a clear tendency to worsen their FF over the time.

Keywords: aging, multimodal training, physical activity, older women, day care

\section{Introduction}

The population is aging, and one of the main consequences of this is the progressive decline in functional fitness (FF), including muscular strength, flexibility, balance, agility, gait velocity, and cardiorespiratory fitness. ${ }^{1} \mathrm{FF}$ is generally defined as the ability to perform daily living activities without difficulty ${ }^{2}$ and represents a powerful and independent risk factor for premature mortality. ${ }^{3}$ However, the decline in FF with aging does not occur at a uniform rate; a significant decrease occurs with advancing age, and the years between 70 and 80 appears to be a critical period of life. ${ }^{4}$

It has been well established that older adults who spend more time in physical activity (PA) or less time in sedentary behaviors exhibit improved FF. Previous studies indicate that physically active older women have performance patterns of flexibility, balance, and agility that are more similar to younger participants than to their older
Parque Desportivo da UTAD, Apartado I0I3, 500I-80I Vila Real, Portugal

Tel +35 I 93640780 I

Fax +35I 259350756

Email nelsons@utad.pt 
inactive peers. ${ }^{5}$ Therefore, it is important for older women to exercise regularly in order to maintain or recover FF. Several studies have shown that different types of exercise interventions in older adults improve flexibility, ${ }^{6}$ strength, ${ }^{7}$ walking performance,${ }^{8}$ postural control, ${ }^{9}$ and most important, muscular fitness, all of which result in better cardiovascular health. ${ }^{10,11}$ Recently, regular multimodal training, based on combined aerobic and resistance exercise, has demonstrated effectiveness in improving FF in older men and women, ${ }^{12,13}$ but to our knowledge, no studies have analyzed the impact of the same training program in different age groups of the elderly population.

Currently available studies suggest that institutionalized elderly people have a low level of PA and spend their time doing little or nothing, which restricts their daily life activities. ${ }^{14}$ In fact, institutionalization has been connected to a significantly lower level of FF. ${ }^{15}$ This is, in fact, evident when institutionalized individuals are compared to their independently living (IL) peers. Several studies from different countries have reported better FF in elderly people living at home than in those in permanent and/or day care (DC)-assisted living facilities. ${ }^{16-19}$ However, the FF levels in permanent DC over time have not yet been investigated.

Thus, in the present study, for analytical reasons as well for practical application, we intend to first compare the FF levels between IL and institutionalized DC elderly women of different age groups. Second, we plan to evaluate the effects of an 8-month multimodal exercise program on the FF of the same IL population. Third, we aim to find out if there is any change in FF levels of institutionalized elderly women after 8 months of permanent DC.

\section{Materials and methods Participants}

A cross-sectional study was conducted with 700 elderly women from social projects of the Rio de Janeiro City Hall, Brazil, including 520 IL subjects and 180 institutionalized DC subjects (care and services provided to elderly adults who require supervision only during the day) aged 60 years and above. All participants were recruited and voluntarily participated in the study. A total of nine institutions (20 participants from each) were selected according to their potential interest in collaborating in the area of FF and PA diagnosis. In each selected institution, a meeting was organized with the management's consent in order to present the aim of the study and to describe the examination procedure in detail. Participants were eligible for the study if they were between 60 years and 79 years of age, had no history of previous exercise training or recreational sports practice, and were not enrolled in any other supervised exercise program. The exclusion criteria included the following: those who smoked; those with diabetes; those with severe obesity; those with severe hypertension; those with a history of falls; those with neurological, mental, or cognitive disorders; those with orthopedic, pulmonary, or cardiac conditions (eg, arrhythmias, history of angina, myocardial infarction, coronary bypass surgery, valvular disease); and those who used mechanical aids for walking.

For analysis of this study, the participants were divided into eight groups according to their ages and social status: IL 60- to 64-year-olds (IL60-64), DC 60- to 64-year-olds (DC60-64), IL 65- to 69-year-olds (IL65-69), DC 65- to 69-year-olds (DC65-69), IL 70- to 74-year-olds (IL70-74), DC 70- to 74-year-olds (DC70-74), IL 75- to 79-year-olds (IL75-79), and DC 75- to 79-year-olds (DC75-79). An exercise program was applied to the IL groups, while in the DC groups, only the institution's activities were considered, including card games, chess, reading, and representation.

Both verbal and written consents were obtained from each participant prior to testing and training; all were informed of the objectives, procedures, and potential risk or discomfort. The study was approved by the University of Castelo Branco review board for human subjects, according to the Declaration of Helsinki.

\section{Exercise program protocol}

The intervention consisted of an 8-month-long multimodal training program with three sessions each week that were planned to be of moderate intensity. According to the latest position stand from the American College of Sports Medicine, moderate intensity represents a perceived exertion of $12-13$ points (Borg scale of 6-20) and a range of 50\%-69\% of one-repetition maximum. ${ }^{20}$

The participants in the IL groups were informed that a minimum of 77 sessions during the 32 weeks ( $80 \%$ compliance) was required to be included in the analysis. Before training, all participants received 2 weeks of familiarization sessions with the training equipment and exercises to be used in the intervention. The multimodal training sessions consisted of the following: (i) a 20-minute warm-up period that included walking (at an intensity perceived as comfortable), and flexibility and balance exercises (eg, one foot support and tandem walking); (ii) a 20-minute cardiorespiratory period, including walking and/or jogging and/or ergometer cycle; (iii) a 30-minute neuromuscular exercise, which included half squat, vertical chest press, back row, abdominal crunch, and lumbar extension exercises (three sets, 15-20 repetitions) using the bodyweight and elastic bands; and (iv) a 5-minute 
cool-down period that included breathing and stretching exercises. All sessions lasted approximately 75 minutes and were always supervised by a professionally qualified instructor. In order to control the target intensity, the values of perceived exertion using the Borg scale were recorded.

\section{Measurements}

Each participant reported to the facility at 7 am on two separate occasions (baseline and after 32 weeks). Height and weight were measured on a standard scale with a stadiometer (SECA 770, Seca Corporation, Hamburg, Germany), and body mass index was calculated using the standard formula: weight $(\mathrm{kg}) /$ height $^{2}(\mathrm{~m})$.

FF was assessed using the Senior Fitness Test battery, which consists of six items designed and validated to assess the physiological parameters that support physical mobility in older adults. ${ }^{21} \mathrm{FF}$ parameters selected were lower- and upper-body strength, lower- and upper-body flexibility, agility/dynamic balance, and aerobic endurance. The items were evaluated using the following tests: 30 -second chair stand (repetitions), arm curl (repetitions), chair sit-and-reach (centimeters), back scratch (centimeters), 8-ft up-and-go (seconds), and 6-minute walk (meters), always in this order. Each test was conducted according to the instructions given by its author(s). Before the test, subjects performed a standard 5- to 10-minute warm-up including stretching exercises. All the tests, except the 6-minute walk test, were performed twice, and the best score was recorded.

\section{Data analysis}

All data analysis was made using the software Statistical Package for Social Sciences version 17.0 (SPSS Inc., Chicago, IL, USA). An exploratory analysis of all data was made to determine the values of the different variables in terms of central tendency and dispersion. An assessment of the normality and sphericity of the distribution of the collected data was applied aiming for an inferential statistical analysis. Thus, a distribution analysis was made using the Kolmogorov-Smirnov test and the Mauchly test.

Once the referred statistical procedures were applied and the conditions for the use of parametric statistics were ensured, $t$-test for independent measures was used to compare means of variables between groups (IL vs DC) at baseline values in all functional tests. Analysis of covariance (ANCOVA) was used to determine whether significant differences existed between pre- and posttest conditions and between the IL and DC groups, with baseline measurements used as covariates in the analysis. The effect size was present by the partial eta square $\left(\eta_{\mathrm{p}}{ }^{2}\right)$, as suggested by Cohen. ${ }^{22}$ The statistical significance was set at $P<0.05$.

\section{Results}

Out of the total number of participants, 26 dropped out, and 15 IL elderly women (3\%) did not complete the study with $80 \%$ compliance as required. A total of eleven institutionalized DC elderly women ( $6 \%$ ) did not complete both assessment moments. A total of 674 participants were included in the analysis. The mean values of age and body mass index of all groups at baseline are shown in Table 1.

At baseline, significant differences were identified between all IL groups and DC groups in the 30-second chair stand, arm curl, back scratch, 8-ft up-and-go, and 6-minute walk $(P<0.001$ for all). Significant differences were identified in IL60-64 vs DC60-64, IL65-69 vs DC65-69, and IL75-79 vs DC75-79 in the chair sit-and-reach $(P<0.001$ for all), but no differences were found in IL70-74 vs DC70-74 $(P=0.178)$ at baseline.

ANCOVA with baseline as covariate identified significant and positive improvements in the 30 -second chair stand, arm curl, back scratch, 8-ft up-and-go, and 6-minute walk between pre- and posttests in all IL groups $(P<0.001$ for all). No differences were found between pre- and posttests in the chair sit-and-reach for the IL70-74 group ( $P=0.211)$. ANCOVA also showed a significant declining performance in the 30-second chair stand, arm curl, back scratch, 8-ft up-and-go, and 6-minute walk between pre- and posttests for all DC groups $(P<0.001$ for all). No differences were found between pre- and posttests in the chair sit-and-reach for the DC70-74 and DC75-79 groups ( $P=0.307$ and $P=0.168$, respectively). Absolute changes during the study period are shown in Table 2. Table 3 shows the effect size in all comparisons performed.

Table I Age and body mass index mean values of all groups at baseline

\begin{tabular}{llll}
\hline & $\mathbf{N}$ & Age (years) & Body mass index $\left(\mathbf{k g} / \mathbf{m}^{2}\right)$ \\
\hline IL60-64 & I49 & $61.9 \pm 1.4$ & $26.9 \pm 3.7$ \\
DC60-64 & 35 & $63.0 \pm 1.2$ & $27.8 \pm 4.6$ \\
IL65-69 & I38 & $66.9 \pm 1.5$ & $27.6 \pm 3.9$ \\
DC65-69 & 34 & $67.8 \pm 1.3$ & $28.4 \pm 3.2$ \\
IL70-74 & I35 & $71.8 \pm 1.5$ & $26.6 \pm 3.8$ \\
DC70-74 & 47 & $72.6 \pm 1.3$ & $28.2 \pm 2.8$ \\
IL75-79 & 83 & $76.7 \pm 1.4$ & $26.8 \pm 4.1$ \\
DC75-79 & 53 & $77.6 \pm 1.2$ & $28.1 \pm 3.8$ \\
\hline
\end{tabular}

Note: Values represent mean \pm standard deviation.

Abbreviations: IL60-64, independent-living 60- to 64-year-olds; DC60-64, day care 60- to 64-year-olds; IL65-69, independent-living 65- to 69-year-olds; DC65-69, day care 65- to 69-year-olds; IL70-74, independent-living 70- to 74-year-olds; DC70-74, day care 70- to 74-year-olds; IL75-79, independent-living 75- to 79-yearolds; DC75-79, day care 75- to 79-year-olds. 

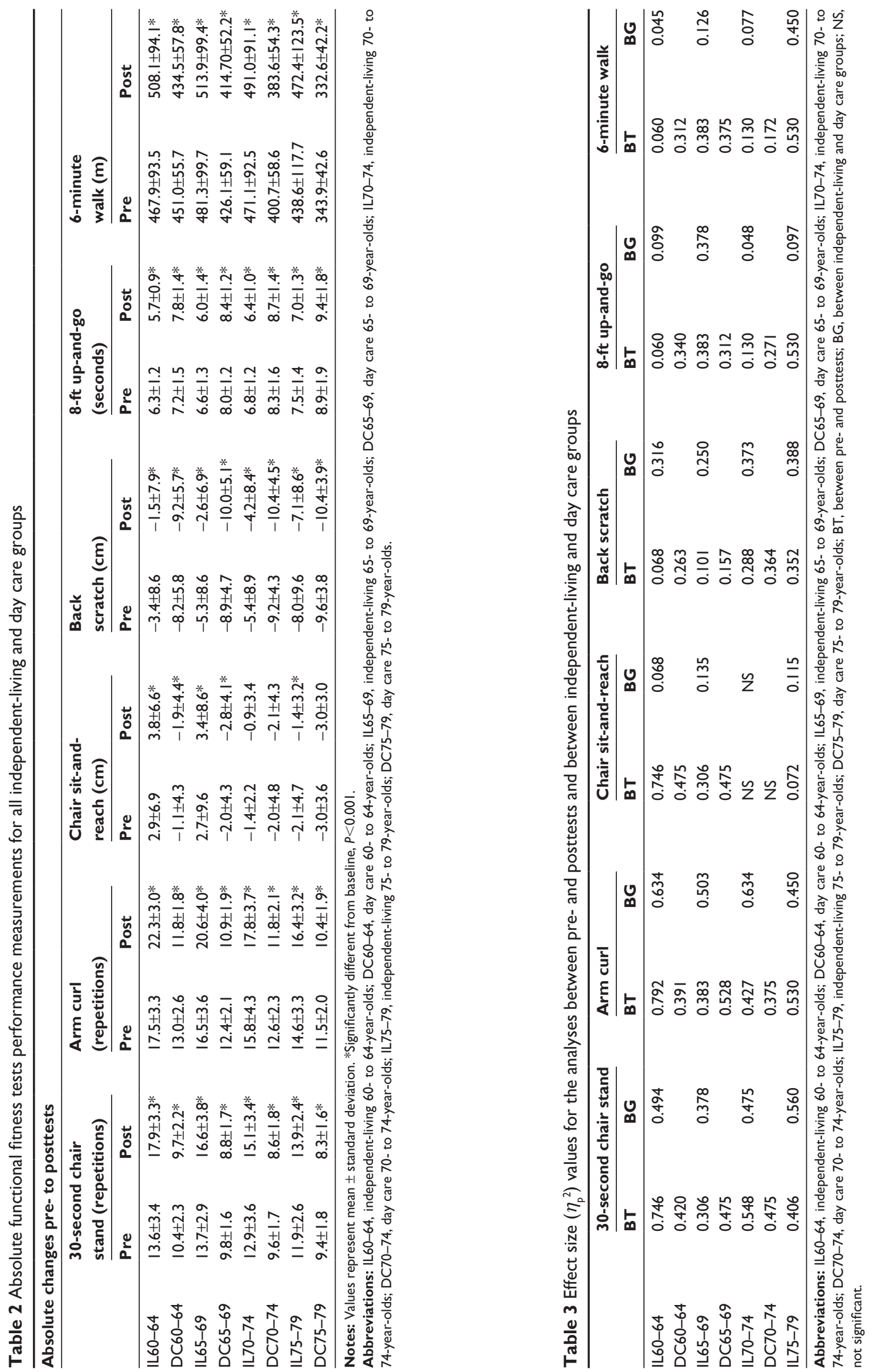


\section{Discussion}

This study confirmed that the IL elderly had a far better functional performance than those in residential DC, as has been observed in previous studies. ${ }^{16-19}$ Cunningham et $\mathrm{al}^{16}$ showed that IL older adults compared to dependent ones showed significantly greater flexibility, activity levels, and walking speed. Another study showed that IL elderly were in better health and had better FF than those in residential care. ${ }^{17}$ Schroeder et $a 1^{18}$ reported that older adults living in several nursing facilities in the US had poorer FF test scores (ie, dynamic balance, leg extension strength, leg press strength, and flexibility) and PA than IL elderly. In the present study, the IL elderly women showed significantly better performance in all components of FF (ie, strength of upper and lower limbs, flexibility of the upper and lower limbs, agility, and aerobic endurance) compared to institutionalized DC elderly women.

Our results suggest that spending the day in care facilities accentuates somewhat the decline in FF. This theory is strengthened by the fact that we found significant differences in FF levels in all age groups, with the only exception in the chair sit-and-reach test, which evaluates the flexibility of the lower limbs, at the ages of 70-75 years. It has been documented that at the age of 71 years, both upper- and lower-body flexibility show an accelerated decline in males, whereas in females, only upper-body flexibility shows a change in the rate of decline, with lower body showing a steady rate of change. ${ }^{23} \mathrm{McCulloch}$ et al ${ }^{24}$ showed little decline in sit-and-reach scores in women vs men, who showed a dramatic decline in age groups of 65-75 years. However, only elderly women were included in the present study. It seems that that the lack of significant differences in the performance of the chair sit-and-reach in those aged 70-75 years could be attributed to regular use of lower-limb joints, for example, those used more in activities of daily living. We believe that independent mobility is probably one of the last daily functions to be lost with aging, and the participants of the present study demonstrated independent mobility.

This study also resulted in notably significant improvements in all FF tests for the IL elderly women. We also observed effectiveness in all age groups, except in the chair sit-and-reach for the 70- to 75-year-old group. Once again, for this age group, we did not find improvements in lower-limb flexibility. In the present study, the training program included several flexibility exercises during the warm-up period, but no specific flexibility training was included in the training sessions, suggesting that the mechanical stimulation of the joints requires more specific exercises. Stathokostas et $\mathrm{a}^{23}$ in a systematic review about flexibility training and functional ability in older adults recommended flexibility-training interventions as supplemental to other forms of exercise, for more effective results. Despite the absence of changes in the latter age group, in the others, there were significant improvements in flexibility, both in the upper and lower limbs. Moreover, flexibility is fundamental to the preservation of functionality, autonomy, quality of life, and well-being. . $5-27^{-2}$

In relation to muscular strength, the multimodal training showed effectiveness in improving both upper- and lowerlimb strength. Muscle strength is the most important predictor of functional capacity for the performance of various activities of daily living in the elderly. ${ }^{13,28}$ Previous studies have showed similar results combining different modes of exercise, with great results increasing both upper- and lowerlimb muscular strength. ${ }^{12,13,29}$ In addition, in the performance of the 8-ft up-and-go, which is a demonstration of agility but is highly dependent on the mechanical power developed by the lower limbs, ${ }^{21}$ significant differences were identified after the multimodal training. Thus, it seems reasonable to believe that the IL elderly women significantly improved the performance of tasks dependent on muscle strength (eg, sitting and rising from a chair, rapid movements, and quick changes in direction), as reported in previous studies where the participants improved their maximum strength. ${ }^{6,30}$

The ability to walk as far as possible in a given amount of time is an important component of quality of life and functionality of older adults. ${ }^{31}$ The results clearly demonstrated that this multimodal training improved endurance. However, we also believe that for this test's characteristics, specifically its short duration, the success of its performance is also dependent on peripheral adaptations, particularly the power and muscle strength of the lower limbs. ${ }^{12}$ Thus, the significant improvements in the 6-minute walk can be, once more, attributed to the improvements in muscular strength.

Similar positive gains in all FF components after a multimodal training intervention have been shown in other studies, ${ }^{13,32-34}$ but no other study tested the effects of the same exercise program in different elderly age groups. Indeed, the improvements reported in the present study were observed in all age groups, from 60 years to 79 years. Our training design very clearly met the minimum standards recommended in guidelines for older individuals. ${ }^{20}$ Generally, multimodal training satisfies all exercise recommendations and represents an effective tool for developing all fitness components.

In contrast, the $\mathrm{DC}$ groups showed a clear tendency to reduce their FF after 8 months, suggesting that institutionalization 
is, indeed, associated with a marked decrease in PA, as previously reported in other studies. ${ }^{13,15}$ The causes may be, in principle, that living conditions in assisted living facilities are often unfavorable for PA and maintaining of FF. ${ }^{15,34}$ The institutionalized elderly women in the present study, during the time that remained in the DC facilities, participated in social activities such as card games, chess, reading, and representation, but none of them involved stimulating PA. Therefore, our results reinforce the importance not only of promoting multimodal exercise training but also of reducing sedentary behaviors in DC institutions for the elderly.

The main limitation of our study is related to its design. The participants were not randomized, and there were no IL control subjects. However, this was a community-based program included in a city hall project. Another limitation of the study in the comparative analysis is the fact that the examined persons in the groups were incomparable in terms of sample (the group of DC participants was much smaller). However, it is very difficult to recruit institutionalized elderly persons who wish to participate voluntarily and who meet all the inclusion criteria. The fact that spontaneous PA was not controlled might also be considered a limitation.

The strength of our study was that only elderly women were included for analysis, and this has been a criterion that has been neglected so far. The long-term intervention with a sample size much larger than that found in the literature and with age-group stratification can also be classified as strengths of this study.

\section{Conclusion}

In conclusion, IL elderly women are more fit than institutionalized DC elderly women.

A multimodal exercise program that combined aerobic and resistance training was effective in improving all FF components related to daily living activities in all age groups except for flexibility of lower limbs in the age group 70-74 years. These results also underline the importance of older people's participation in regular physical exercise for their health and quality of life.

Finally, institutionalized elderly women showed a clear tendency to reduce their FF over time. Professionals should consider the importance of preserving functional capacity by recommending regular PA and the reduction of sedentary behaviors in DC living facilities.

\section{Acknowledgment}

We express our deep appreciation to all of the participants and senior citizen volunteers of Rio de Janeiro city.

\section{Disclosure}

The authors report no conflicts of interest in this work.

\section{References}

1. Buchman A, Boyle P, Wilson R, Bienias J, Bennett D. Physical activity and motor decline in older persons. Muscle Nerve. 2007;35(3): 354-362.

2. Rikli RE, Jones CJ. Development and validation of criterion-referenced clinically relevant fitness standards for maintaining physical independence in later years. Gerontologist. 2013;53(2):255-267.

3. Church TS, Earnest CP, Skinner JS, Blair SN. Effects of different doses of physical activity on cardiorespiratory fitness among sedentary, overweight or obese postmenopausal women with elevated blood pressure: a randomized controlled trial. JAMA. 2007;297(19): 2081-2091.

4. Demura S, Minami M, Nagasawa Y, Tada N, Matsuzawa J, Sato S. Physical-fitness declines in older Japanese adults. J Aging Phys Act. 2003; 11:112-122.

5. Rikli RE, Busch S. Motor performance of women as a function of age and physical activity level. J Gerontol. 1986;41:645-649.

6. Stathokostas L, Little RM, Vandervoort AA, Paterson DH. Flexibility training and functional ability in older adults: a systematic review. J Aging Res. 2012;2012:306818.

7. Sousa N, Sampaio J. Effects of progressive strength training on the performance of the Functional Reach Test and the Timed Get-Upand-Go Test in an elderly population from the rural north of Portugal. Am J Hum Biol. 2005;17(6):746-751.

8. Malatesta D, Simar D, Ben Saad H, Préfaut C, Caillaud C. Effect of an overground walking training on gait performance in healthy 65 - to 80-year-olds. Exp Gerontol. 2010;45(6):427-434.

9. Keogh JW, Morrison S, Barrett R. Strength and coordination training are both effective in reducing the postural tremor amplitude of older adults. J Aging Phys Act. 2010;18(1):43-60.

10. Artero EG, Lee DC, Lavie CJ, et al. Effects of muscular strength on cardiovascular risk factors and prognosis. J Cardiopulm Rehabil Prev. 2012;32(6):351-358.

11. Menezes AR, Lavie CJ, Forman DE, Arena R, Milani RV, Franklin BA. Cardiac rehabilitation in the elderly. Prog Cardiovasc Dis. 2014;57(2): 152-159.

12. Sousa N, Mendes R, Abrantes C, Sampaio J, Oliveira J. Effectiveness of combined exercise training to improve functional fitness in older adults: a randomized controlled trial. Geriatr Gerontol Int. 2014;14: 892-898.

13. Sousa N, Mendes R. Effects of resistance versus multicomponent training on body composition and functional fitness in institutionalized elderly women. J Am Geriatr Soc. 2013;61(10):1815-1817.

14. Ice GH. Daily life in a nursing home: has it changed in 25 years? J Aging Stud. 2002;16:345-359.

15. Król-Zielinska M, Kusy K, Zielinski J, Osiński W. Physical activity and functional fitness in institutionalized vs independently living elderly: a comparison of 70-80-year-old city-dwellers. Arch Gerontol Geriatr. 2011; 53:e10-e16.

16. Cunningham DA, Paterson DH, Himann JE, Rechnitzer PA. Determinants of independence in the elderly. Can J Appl Physiol. 1993;18: 243-254.

17. Noro A, Aro S. Comparison of health and functional ability between noninstitutionalized and least dependent institutionalized elderly in Finland. Gerontologist. 1997;37:374-383.

18. Schroeder JM, Nau KL, Osness H, Potteiger JA. A comparison of life satisfaction, functional ability, physical characteristics, and activity level among older adults in various living settings. J Aging Phys Act. 1998; 6:340-349.

19. Dobrzyn-Matusiak D, Marcisz C, Bąk E, Kulik H, Marcisz E. Physical and mental health aspects of elderly in social care in Poland. Clin Interv Aging. 2014;21(9):1793-1802. 
20. Garber CE, Blissmer B, Deschenes MR, et al. American College of Sports Medicine position stand. Quantity and quality of exercise for developing and maintaining cardiorespiratory, musculoskeletal, and neuromotor fitness in apparently healthy adults: guidance for prescribing exercise. Med Sci Sports Exerc. 2011;43(7):1334-1359.

21. Rikli RE, Jones CJ. Development and validation of a functional fitness test for community-residing older adults. J Aging Phys Act. 1999;7:129-161.

22. Cohen J. Statistical Power Analysis for the Behavioral Sciences. 2nd ed. New York: Routledge; 2013.

23. Stathokostas L, McDonald MW, Little RM, Paterson DH. Flexibility of older adults aged 55-86 years and the influence of physical activity. J Aging Res. 2013;2013:743843.

24. McCulloch RG, Clark DJ, Pike I, Slobodian YM. Gender specific trends in fitness and anthropometric parameters in a selected Saskatchewan sample, aged 65-75 years. Can J Aging. 1994;13(1):30-40.

25. Orr R, Raymond J, Fiatarone Singh M. Efficacy of progressive resistance training on balance performance in older adults: a systematic review of randomized controlled trials. Sports Med. 2008;38(4):317-343.

26. Seco J, Abecia LC, Echevarría E, et al. A long-term physical activity training program increases strength and flexibility, and improves balance in older adults. Rehabil Nurs. 2013;38:37-47.

27. Battaglia G, Bellafiore M, Caramazza G, Paoli A, Bianco A, Palma A. Changes in spinal range of motion after a flexibility training program in elderly women. Clin Interv Aging. 2014;9:653-660.
28. Misic MM, Rosengren KS, Woods JA, Evans EM. Muscle quality, aerobic fitness and fat mass predict lower-extremity physical function in community-dwelling older adults. Gerontology. 2007;53(5):260-266.

29. Gudlaugsson J, Gudnason V, Aspelund T, et al. Effects of a 6-month multimodal training intervention on retention of functional fitness in older adults: a randomized-controlled cross-over design. Int J Behav Nutr Phys Act. 2012;9:107.

30. Capodaglio P, Capodaglio Edda M, Facioli M, Saibene F. Long-term strength training for community-dwelling people over 75: impact on muscle function, functional ability and life style. Eur J Appl Physiol. 2007; 100(5):535-542.

31. Enright PL, McBurnie MA, Bittner V, et al; Cardiovascular Health Study. The 6-min walk test: a quick measure of functional status in elderly adults. Chest. 2003;123(2):387-398.

32. Toraman NF, Erman A, Agyar E. Effects of multicomponent training on functional fitness in older adults. J Aging Phys Act. 2004;12: 538-553.

33. Carvalho MJ, Marques E, Mota J. Training and detraining effects on functional fitness after a multicomponent training in older women Gerontology. 2009;55:41-48.

34. De Lange E, Van der Veen WJ, Van der Werf GT. Primary care in homes for the elderly. Tijdschr Gerontol Geriatr. 2008;39:107-114.
Clinical Interventions in Aging

\section{Publish your work in this journal}

Clinical Interventions in Aging is an international, peer-reviewed journal focusing on evidence-based reports on the value or lack thereof of treatments intended to prevent or delay the onset of maladaptive correlates of aging in human beings. This journal is indexed on PubMed Central, MedLine,

\section{Dovepress}

CAS, Scopus and the Elsevier Bibliographic databases. The manuscript management system is completely online and includes a very quick and fair peer-review system, which is all easy to use. Visit http://www.dovepress. com/testimonials.php to read real quotes from published authors. 